Research Article

\title{
On the Motion of Two Microspheres in a Stokes Flow Driven by an External Oscillator Field
}

\author{
Mohammed M. Al-Hatmi $(\mathbb{D})$ and Anton Purnama \\ Department of Mathematics, College of Science, Sultan Qaboos University, Muscat, Oman \\ Correspondence should be addressed to Mohammed M. Al-Hatmi; s91615@student.squ.edu.om
}

Received 16 June 2021; Accepted 21 October 2021; Published 22 November 2021

Academic Editor: Remi Léandre

Copyright (c) 2021 Mohammed M. Al-Hatmi and Anton Purnama. This is an open access article distributed under the Creative Commons Attribution License, which permits unrestricted use, distribution, and reproduction in any medium, provided the original work is properly cited.

\begin{abstract}
Hydrodynamic interactions of a two-solid microspheres system in a viscous incompressible fluid at low Reynolds number is investigated analytically. One of the spheres is conducting and assumed to be actively in motion under the action of an external oscillator field, and as the result, the other nonconducting sphere moves due to the induced flow oscillation of the surrounding fluid. The fluid flow past the spheres is described by the Stokes equation and the governing equation in the vector form for the twosphere system is solved asymptotically using the two-timing method. For illustrations, applying a simple oscillatory external field, a systematic description of the average velocity of each sphere is formulated. The trajectory of the sphere was found to be inversely proportional to the frequency of the external field. The results demonstrated that no collisions occur between the spheres as the system moves in a circular motion with a fixed separation distance.
\end{abstract}

\section{Introduction}

Dynamics of microparticles in a viscous fluid play important roles in many applications in medicine and technology, such as minimising surgical invasion and controlling drug delivery, see, e.g., [1] and [2]. The study of the motion of small particles in suspension has been of interest to scientists for many years and is still an active area of research, see, e.g., [3-8]. The movement of a particle due to the oscillatory external forces in a viscous fluid represents a classical problem of fluid dynamics. This motion represents a model problem for the use of the dynamical approach in fluid dynamics and for the studies of turbulence.

A conducting or active microsphere suspended in an external oscillatory force tends to accelerate in the direction of the applied external field. Although this type of interaction is classical in character, there are certain features that do not seem to be widely understood. Interactions of a system of two microspheres are two-fold: first, the active sphere moves in the direction of the external field, and second, the other nonconducting sphere moves due to the local surrounding fluid velocity generated by the motion of the active sphere. There is, however, a secondary effect arising from the fact that the active sphere has not solely moved due to the external field, but its motion is also distorted by the presence of the other sphere. The key question is how does the surrounding fluid influence the motion of a nonconducting sphere; will it also change the direction the active sphere moves, and if so, what trajectory does the active sphere follow? Does the size of the microspheres have any effects on the motion of the active sphere? In an attempt to answer these questions, we develop an analytical framework to study the motion of a system of two microspheres in Stokes flow driven by an external oscillatory force. This analytical theory serves as a preliminary investigation on the effect of an external oscillatory field on the motion of a suspension of conducting particles.

The purpose of this paper is to provide a systematic and explicit description of the interaction of two rigid microspheres of radii $R_{v}<10^{-6}$ meter in a viscous incompressible fluid and to determine their average velocities. This is a highfrequency asymptotic problem at low Reynolds number, $\operatorname{Re} \ll 1$, powered by an external oscillator field. We consider that one sphere is nonconducting and passive and the other 
is conducting and active which is accelerated by an external oscillatory field such as a magnetic field, see, e.g., $[6,9-11]$ an electric field, see, e.g., [12]; or molecular Brownian forces, see, e.g., [13]. The asymptotic formulation of the problem leads us to study the motion of the spheres with a timeperiodic external force, as described by the Stokes equation, where the fluid inertial effects are neglected. We solved the resulting equations of successive approximations by employing the two-timing method, see, e.g., [14]. We present our results in the general vector form and discuss them in details through an example. Our analytical treatments are simple, but it can be considered as the basis for the development of a full theory of particle suspension.

\section{Formulation of Problem}

We consider the motion of a system of two microspheres of equal density and different radii $R_{v}$ in a viscous incompressible quiescent fluid at low Reynolds number. One of the spheres $\mathbf{x}^{(1)}$ is conducting and actively moving under the influence of an external field which oscillates periodically with constant frequency $\omega$, and the other $\mathbf{x}^{(2)}$ is nonconducting and its movement is due to the fluid disturbance generated by the first sphere. In the Cartesian coordinates, the position vector of the centre of the microspheres and the unit vector $\mathbf{n}$ along the displacement vector $\mathbf{r}$ are described by

$$
\mathbf{x}^{(v)}=\left(x_{1}^{(\nu)}, x_{2}^{(v)}, x_{3}^{(v)}\right), \quad \mathbf{n} \equiv \frac{\mathbf{r}}{r}, \mathbf{r}=\mathbf{x}^{(1)}-\mathbf{x}^{(2)},
$$

where the distance $r=|\mathbf{r}|$ is relatively large in comparison with $R_{v}$, see Figure 1 . We use the subscripts $i, j, k=1,2,3$ for the Cartesian components of the vectors and tensors and superscripts $\mu, \nu=1,2$ for the spheres.

The oscillatory motions of the microspheres are prescribed as a three-dimensional transitional spatial displacement vector $\tilde{\xi}(\tau)=\left(\xi_{1}(\tau), \xi_{2}(\tau), \xi_{3}(\tau)\right), \quad \tau=\omega t$, through the related induced acceleration $\widetilde{\xi}_{t t}=\omega^{2} \widetilde{\xi}_{\tau \tau}$, where the subscripts $t$ and $\tau$ stand for the associated derivatives. This problem is classified as an oscillating (noninertial) system of reference, in which a fluid at infinity is termed in a state of rest. In this frame of motion, according to Einstein's principle of equivalence which states that there is an equivalence of gravitational and inertial mass, there is no influence of gravity field on the microspheres, and thus the equations of fluid motion are standard, see, e.g., [15]. Hence, the microspheres float in the fluid due to the buoyancy force

$$
\mathbf{f}_{b}^{(\nu)}=-M^{(\nu)} \widetilde{\mathbf{g}}(\tau),
$$

where $M=(-4 / 3) \pi R_{v}^{3}\left(\rho_{p}-\rho_{f}\right) ; \rho_{p}$ is the density of the microsphere; $\rho_{f}$ the density of the fluid; and $\widetilde{\mathbf{g}}(\tau)$ is equivalent to the induced acceleration

$$
\widetilde{\mathbf{g}}(\tau)=\omega^{2} \widetilde{\boldsymbol{\xi}}_{\tau \tau}
$$

Throughout the paper, a 'tilde' above a function denotes a $2 \pi$-periodic function with zero mean value.

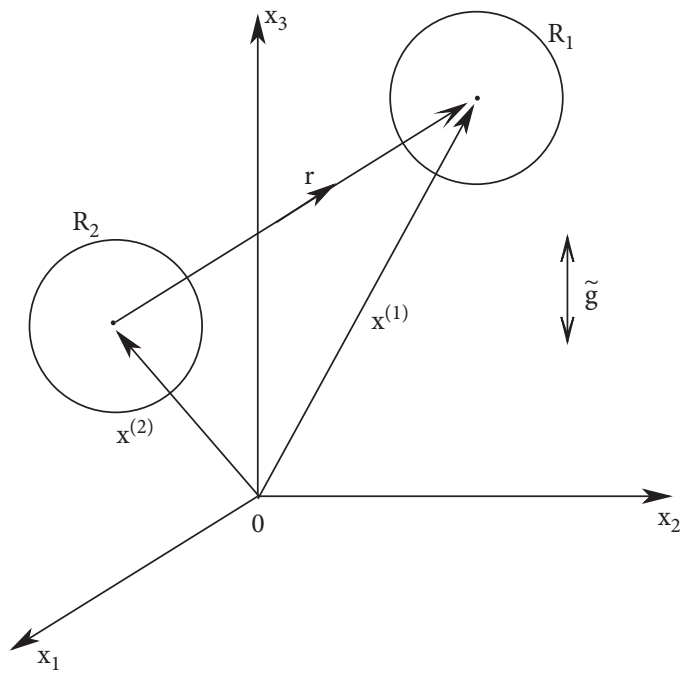

FIgURE 1: Diagram of a system of two microspheres in a viscous fluid.

We consider the separation distance $r$ between the centres of the two microspheres which is less than the thickness of the Stokes boundary layer $(v / \omega)^{1 / 2}$, where $v$ is the kinetic viscosity of the fluid. Hence, the explicit expressions for the Stokes friction force $\mathbf{f}_{h}^{(\nu)}$ exerted on each sphere during the motion, see. e.g., [16], is defined as

$$
\mathbf{f}_{h}^{(v)}=6 \pi \eta R_{\nu}\left(\mathbf{x}_{t}^{(\nu)}-\mathbf{u}_{\mu \nu}\right), \quad \mu \neq \nu,
$$

where $\eta$ is the dynamic viscosity of the fluid and $\mathbf{u}$ is the induced velocity due to the movement of the other sphere, see, e.g., [2], defined as

$$
\mathbf{u}_{\mu \nu}=\frac{3 R_{\mu}}{4}\left(\frac{\mathbf{x}_{t}^{(\mu)}+\mathbf{n}\left(\mathbf{x}_{t}^{(\mu)} \cdot \mathbf{n}\right)}{r}\right),
$$

such that $\mathbf{u}_{12}$ represents the velocity generated by the movement of the first sphere on the second, and similarly, $\mathbf{u}_{21}$ is the velocity due to the movement of the second sphere on the first.

The fluid flow past the microspheres is described by the Stokes equation

$$
\mathbf{f}_{h}^{(v)}+\mathbf{f}_{b}^{(v)}=0
$$

where all inertial terms are neglected. The use of (2) and (4) and (5) into (6) yields the equation of motion:

$$
6 \pi \eta R_{\nu}\left(\mathbf{x}_{t}^{(\nu)}-\frac{3 R_{\mu}}{4 r}\left(\mathbf{x}_{t}^{(\mu)}+\mathbf{n}\left(\mathbf{x}_{t}^{(\mu)} \cdot \mathbf{n}\right)\right)\right)=M^{(\nu)} \widetilde{\mathbf{g}} .
$$

As shown in Figure 1, the geometric configuration of the problem contains two characteristic lengths: the distance between the centres of microspheres $L$ and the mean radius of the microspheres $R$. For illustrations, we choose the following reference scales: 


$$
\begin{aligned}
R & =\frac{R_{1}+R_{2}}{2}, \\
T & =\frac{L}{U}, \\
G & =\max |\widetilde{\mathbf{g}}(\tau)|, \\
F & =\frac{6 \pi \eta R L}{T},
\end{aligned}
$$

where $T, G, M$, and $F$ are the characteristic time, magnitudes of induced acceleration, mass, and Stokes friction force, respectively. The dimensionless variables (marked with asterisks) are chosen as

$$
\begin{aligned}
x & =x^{*} L, \\
R_{v} & =R R_{v}^{*}, \\
t & =T t^{*}, \\
\widetilde{\mathbf{g}} & =G \widetilde{\mathbf{g}}^{*}, \\
f_{h}^{(v)} & =F f_{h}^{*(v)}, \\
\omega^{*} & =\omega T .
\end{aligned}
$$

Three independent small parameters of the problem are

$$
\begin{aligned}
& \epsilon=\frac{1}{\omega^{*}}, \\
& \delta=\frac{3 R}{4 L}, \\
& m=\frac{M G}{F} ; \quad \epsilon, \delta \ll 1 .
\end{aligned}
$$

Substituting (8)-(10) into (7), and after simplification, yields the dimensionless form (all asterisks are omitted for brevity) of the equation

$$
\mathbf{x}_{t}^{(\nu)}-\delta R_{\mu} \mathbb{S} \mathbf{x}_{t}^{(\mu)}=m R_{\nu}^{2} \widetilde{\mathbf{g}}(\tau), \quad \mu \neq \nu,
$$

where

$$
\mathbb{S}=\mathbb{S}_{i j}(\mathbf{r}) \equiv \frac{1}{r}\left[\delta_{i j}+\mathbf{n}_{i} \mathbf{n}_{j}\right]
$$

where $\quad m=(M G / F)=\left(\left(2 R^{2}\left(\rho_{p}-\rho_{f}\right) G\right) / 9 \eta U\right)=$ const $=$ $O(1)$ and $\delta_{i j}$ is the Kronecker delta; $\delta_{i j}=1$ when $i=j$ and $\delta_{i j}=0$ when $i \neq j$.

An approximate solution to equation (11) is presented in the next section using the two-timing method, an asymptotic procedure involving fast and slow times.

\section{Two-Timing Method and Asymptotic Procedure}

3.1. Definition and Asymptotic Procedure. Two-timing method constructs an asymptotic solution to the equation of motion (11) by introducing two dependent time scales $s$ and $\tau$ as mutually independent variables, called slow and fast times, respectively, see Figure 2. This method converts an

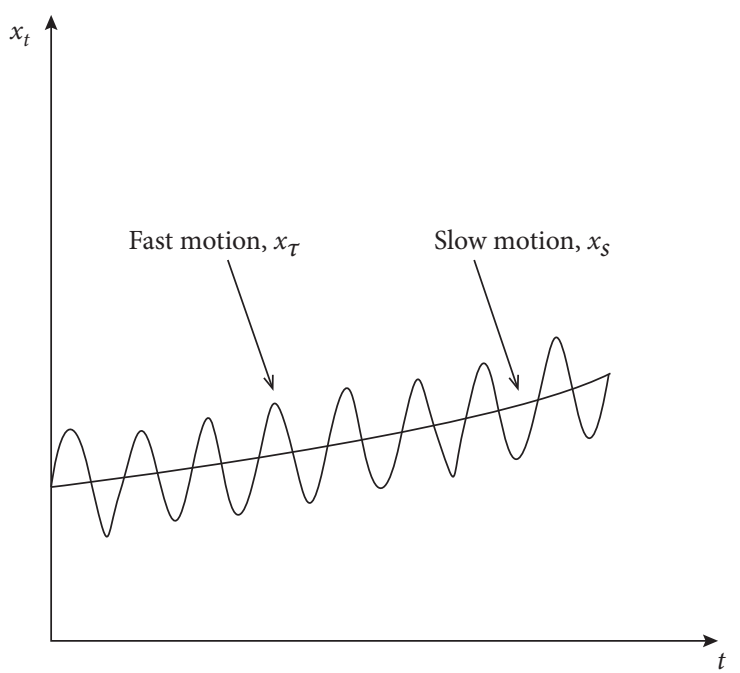

FIGURE 2: Diagram of the fast and slow motions.

ODE (11) with one independent variable $t$ into a PDE with two independent variables $s$ and $\tau$.

The proper relations between $s, \tau$, and $t$, see, e.g., [17], are defined by

$$
\begin{gathered}
\tau=\omega t, \\
s=\frac{t}{\omega^{\alpha}}, \\
\alpha>-1,
\end{gathered}
$$

so that a given value $\alpha$ will lead to a path for the asymptotic solution. In a rigorous asymptotic procedure as $\omega \longrightarrow \infty$, there is a unique path that leads to a valid solution. If such a limit brings valid an asymptotic result, then it is called a distinguished limit. In this paper, we have chosen $\alpha=1$; hence, the two time scales,

$$
\begin{gathered}
\tau=\omega t, \\
s=\frac{t}{\omega},
\end{gathered}
$$

will lead to a valid solution through an asymptotic procedure of successive approximations. This choice can be justified by the distinguished limit arguments as in [14].

3.2. Functions and Notation. For making further progress analytically, we introduce a few convenient notations. We assume that any dimensionless function $f(s, \tau)$, which represents either a scalar, vector, or tensor, see, e.g., [14], has the following properties:

(i) Subscripts $\tau$ and $s$ stand for the related partial time derivatives.

(ii) $f=f(s, \tau)$ belongs to class $O(1)$ such that $f=O(1)$, and all partial $s$ and $\tau$ derivatives of $f$ (required for our consideration) are also $O(1)$. 
(iii) We consider only periodic function in $\tau$ $\{f \in \mathscr{P}: f(s, \tau)=f(s, \tau+2 \pi)\} \quad$ where $s$-dependence is not specified.

(iii) For arbitrary $f \in \mathscr{P}$, the averaging-operation is

$$
\langle f\rangle \equiv \frac{1}{2 \pi} \int_{\tau_{0}}^{\tau_{0}+2 \pi} f(s, \tau) \mathrm{d} \tau \equiv \bar{f}(s), \quad \forall \tau_{0} .
$$

(iv) The bar-function $\bar{f}=\bar{f}(s)$ (or mean-function) does not depend on $\tau$.

(v) The tilde-function, $\tilde{f}(\tau)$ (or purely oscillating function), represents a special case of $\mathscr{P}$-function with zero average

$$
\langle\tilde{f}\rangle=0 .
$$

A unique decomposition is valid

$$
f=\bar{f}+\tilde{f} .
$$

(vi) $\underset{\sim}{\text { A special notation }} \tilde{f}^{\tau}$ is the tilde-integration of $\widetilde{f}(\tau)$,

$$
\tilde{f}^{\tau}=\int_{0}^{\tau} \tilde{f}(s, \sigma) \mathrm{d} \sigma-\frac{1}{2 \pi} \int_{0}^{2 \pi}\left(\int_{0}^{\mu} \tilde{f}(s, \sigma) \mathrm{d} \sigma\right) \mathrm{d} \mu .
$$

The tilde-integration is the inverse of $\tau$-differentiation

$$
\left(\tilde{f}^{\tau}\right)_{\tau} \equiv\left(\tilde{f}_{\tau}\right)^{\tau} \equiv \tilde{f}
$$

3.3. Successive Approximations. The choice (14) of $\tau=\omega t$ and $s=t / \omega$ leads to the following derivative:

$$
\begin{aligned}
\frac{\mathrm{d}}{\mathrm{d} t} & =\omega \frac{\partial}{\partial \tau}+\frac{1}{\omega} \frac{\partial}{\partial s} \\
& =\omega\left(\frac{\partial}{\partial \tau}+\epsilon^{2} \frac{\partial}{\partial s}\right) ; \quad \epsilon=\frac{1}{\omega} .
\end{aligned}
$$

This suggests that we should consider a series of expansions in $\epsilon$ terms to at most $O\left(\epsilon^{2}\right)$ and keeping at most linear in $\delta$ terms. The unknown $\mathbf{x}^{(v)}$ is therefore written as

$$
\begin{aligned}
\mathbf{x}^{(\nu)}(s, \tau)= & \left(\overline{\mathbf{x}}_{0}^{(v)}+\widetilde{\mathbf{x}}_{0}^{(\nu)}\right)+\varepsilon\left(\overline{\mathbf{x}}_{1}^{(\nu)}+\widetilde{\mathbf{x}}_{1}^{(\nu)}\right) \\
& +\varepsilon^{2}\left(\overline{\mathbf{x}}_{2}^{(\nu)}+\widetilde{\mathbf{x}}_{2}^{(\nu)}\right)+O\left(\epsilon^{2}\right) .
\end{aligned}
$$

Differentiating (21) with respect to $t$ gives

$\mathbf{x}_{t}^{(\nu)}=\left(\overline{\mathbf{x}}_{0 t}^{(\nu)}+\widetilde{\mathbf{x}}_{0 t}^{(\nu)}\right)+\varepsilon\left(\overline{\mathbf{x}}_{1 t}^{(\nu)}+\widetilde{\mathbf{x}}_{1 t}^{(\nu)}\right)+\varepsilon^{2}\left(\overline{\mathbf{x}}_{2 t}^{(\nu)}+\widetilde{\mathbf{x}}_{2 t}^{(\nu)}\right)+O\left(\epsilon^{2}\right)$.

The use of (20) into (22) yields to

$$
\begin{aligned}
\mathbf{x}_{t}^{(\nu)}= & \omega\left(\left(\overline{\mathbf{x}}_{0 \tau}^{(\nu)}+\epsilon^{2} \overline{\mathbf{x}}_{0 s}^{(v)}\right)+\left(\widetilde{\mathbf{x}}_{0 \tau}^{(\nu)}+\epsilon^{2} \widetilde{\mathbf{x}}_{0 s}^{(\nu)}\right)\right) \\
& +\omega \epsilon\left(\left(\overline{\mathbf{x}}_{1 \tau}^{(\nu)}+\cdots\right)+\left(\widetilde{\mathbf{x}}_{1 \tau}^{(\nu)}+\cdots\right)\right) \\
& +\omega \epsilon^{2}\left(\left(\overline{\mathbf{x}}_{2 \tau}^{(\nu)}+\cdots\right)+\left(\widetilde{\mathbf{x}}_{2 \tau}^{(\nu)}+\cdots\right)\right)+\cdots
\end{aligned}
$$

The two-timing method studies only the class of solutions with

$$
\widetilde{\mathbf{x}}_{0}(s, \tau)=0, \quad \text { while } \bar{x}_{0}(s, \tau) \neq 0 .
$$

Physically, this constraint means that the amplitude of oscillations is small compared with the amplitude of the averaged solution such that when $\widetilde{\mathbf{x}}_{0}(s, \tau) \neq 0$, the main term of velocity grows to infinity as $\omega \longrightarrow \infty$.

Hence, (23) can be simplified in the form

$$
\mathbf{x}_{t}^{(\nu)}=\omega\left(\epsilon \widetilde{\mathbf{x}}_{1 \tau}^{(\nu)}+\epsilon^{2}\left(\widetilde{\mathbf{x}}_{2 \tau}^{(\nu)}+\overline{\mathbf{x}}_{0 s}^{(\nu)}\right)+O\left(\epsilon^{2}\right)\right) .
$$

Next, Taylor series expansion of the tensor $\mathbb{S}_{i j}$ in (12) about $\mathbf{r}=\mathbf{r}_{0}$ takes the form

$$
\mathbb{S}_{i j}(\mathbf{r})=\mathbb{S}_{i j}\left(\mathbf{r}_{0}\right)+\mathbb{S}_{i j}^{\prime}\left(\mathbf{r}_{0}\right)\left(\mathbf{r}-\mathbf{r}_{0}\right)+O\left(\epsilon^{2}\right),
$$

where $\mathbf{r}-\mathbf{r}_{0}=\epsilon \mathbf{r}_{1}+\epsilon^{2} \mathbf{r}_{2}+O\left(\epsilon^{2}\right)$. Hence, (26) can be simplified and written as

$$
\mathbb{S}_{i j}(\mathbf{r})=\mathbb{S}_{i j}\left(\mathbf{r}_{0}\right)+\epsilon \mathbf{r}_{1 k} \frac{\partial \mathbb{S}_{i j}\left(\mathbf{r}_{0}\right)}{\partial x_{k}}+O\left(\epsilon^{2}\right),
$$

where $\mathbf{r}_{0}, \mathbf{r}_{1 k}, \mathbb{S}_{i j}\left(\mathbf{r}_{0}\right)$, and $\partial \mathbb{S}_{i j}\left(\mathbf{r}_{0}\right) / \partial x_{k}$ are given by

$$
\begin{aligned}
& \mathbf{r}_{0}=\mathbf{x}_{0}^{(1)}-\mathbf{x}_{0}^{(2)}, \\
& \mathbf{r}_{1 k}=\mathbf{x}_{1 k}^{(1)}-\mathbf{x}_{1 k}^{(2)}, \\
& \mathbb{S}_{i j}\left(\mathbf{r}_{0}\right)=\frac{1}{\mathbf{r}_{0}}\left[\delta_{i j}+\mathbf{n}_{0 i} \mathbf{n}_{0 j}\right], \quad \mathbf{n}_{0}=\frac{\mathbf{r}_{0}}{r_{0}} ; r_{0}=\left|\mathbf{r}_{0}\right|, \\
& \frac{\partial \mathbb{S}_{i j}\left(\mathbf{r}_{0}\right)}{\partial \mathbf{x}_{k}}=\frac{1}{\mathbf{r}_{0}^{2}}\left(-\delta_{i j} \mathbf{n}_{0 k}+\delta_{i k} \mathbf{n}_{0 j}+\delta_{j k} \mathbf{n}_{0 i}-3 \mathbf{n}_{0 i} \mathbf{n}_{0 j} \mathbf{n}_{0 k}\right) .
\end{aligned}
$$

For the future use, we write the first and second terms in (27) as

$$
\begin{aligned}
& \mathbb{S}_{0}=\mathbb{S}_{i j}\left(\mathbf{r}_{0}\right) \\
& \mathbb{S}_{1}=\mathbb{S}_{1 i j}\left(\mathbf{r}_{0}\right)=\mathbf{r}_{1 k} \frac{\partial \mathbb{S}_{i j}\left(\mathbf{r}_{0}\right)}{\partial x_{k}}
\end{aligned}
$$

Substituting (25) and (27) into the equation of motion (11) yields

$$
\begin{gathered}
\epsilon \widetilde{\mathbf{x}}_{1 \tau}^{(1)}+\epsilon^{2}\left(\widetilde{\mathbf{x}}_{2 \tau}^{(1)}+\overline{\mathbf{x}}_{0 s}^{(1)}\right)+O\left(\epsilon^{2}\right)-\delta R_{2}\left(\mathbb{S}_{0}+\epsilon \mathbb{S}_{1}+O\left(\epsilon^{2}\right)\right) \\
\cdot\left(\epsilon \widetilde{\mathbf{x}}_{1 \tau}^{(2)}+\epsilon^{2}\left(\widetilde{\mathbf{x}}_{2 \tau}^{(2)}+\overline{\mathbf{x}}_{0 s}^{(2)}\right)+O\left(\epsilon^{2}\right)\right)=\epsilon m R_{1}^{2} \widetilde{\mathbf{g}}
\end{gathered}
$$

$$
\begin{gathered}
\epsilon \widetilde{\mathbf{x}}_{1 \tau}^{(2)}+\epsilon^{2}\left(\widetilde{\mathbf{x}}_{2 \tau}^{(2)}+\overline{\mathbf{x}}_{0 s}^{(2)}\right)+O\left(\epsilon^{2}\right)-\delta R_{1}\left(\mathbb{S}_{0}+\epsilon \mathbb{S}_{1}+O\left(\epsilon^{2}\right)\right) \\
\cdot\left(\epsilon \widetilde{\mathbf{x}}_{1 \tau}^{(1)}+\epsilon^{2}\left(\widetilde{\mathbf{x}}_{2 \tau}^{(1)}+\overline{\mathbf{x}}_{0 s}^{(1)}\right)+O\left(\epsilon^{2}\right)\right)=\epsilon m R_{2}^{2} \widetilde{\mathbf{g}}
\end{gathered}
$$

The successive approximations of (30) and (31) lead to the following:

The terms of order $\epsilon^{0}$ give the identity $0=0$. The terms of order $\epsilon$ yield the equations 


$$
\begin{aligned}
& \widetilde{\mathbf{x}}_{1 \tau}^{(1)}-\delta R_{2} \mathbb{S}_{0} \widetilde{\mathbf{x}}_{1 \tau}^{(2)}=m R_{1}^{2} \widetilde{\mathbf{g}}, \\
& \widetilde{\mathbf{x}}_{1 \tau}^{(2)}-\delta R_{1} \mathbb{S}_{0} \widetilde{\mathbf{x}}_{1 \tau}^{(1)}=m R_{2}^{2} \widetilde{\mathbf{g}} .
\end{aligned}
$$

Integrating (32) using the properties of periodic functions, and keeping at most linear in $\delta$ terms, yields

$$
\begin{aligned}
& \widetilde{\mathbf{x}}_{1}^{(1)}=m\left(\delta R_{2}^{3} \mathbb{S}_{0}+R_{1}^{2}\right) \widetilde{\mathbf{g}}^{\tau}, \\
& \widetilde{\mathbf{x}}_{1}^{(2)}=m\left(\delta R_{1}^{3} \mathbb{S}_{0}+R_{2}^{2}\right) \widetilde{\mathbf{g}}^{\tau} .
\end{aligned}
$$

Next, the terms of order $\epsilon^{2}$ give the equations

$$
\begin{aligned}
& \widetilde{\mathbf{x}}_{2 \tau}^{(1)}+\overline{\mathbf{x}}_{0 s}^{(1)}-\delta R_{2}\left(\mathbb{S}_{0}\left(\widetilde{\mathbf{x}}_{2 \tau}^{(2)}+\overline{\mathbf{x}}_{0 s}^{(2)}\right)+\mathbb{S}_{1} \widetilde{\mathbf{x}}_{1 \tau}^{(2)}\right)=0, \\
& \widetilde{\mathbf{x}}_{2 \tau}^{(2)}+\overline{\mathbf{x}}_{0 s}^{(2)}-\delta R_{1}\left(\mathbb{S}_{0}\left(\widetilde{\mathbf{x}}_{2 \tau}^{(1)}+\overline{\mathbf{x}}_{0 s}^{(1)}\right)+\mathbb{S}_{1} \widetilde{\mathbf{x}}_{1 \tau}^{(1)}\right)=0 .
\end{aligned}
$$

Applying the averaging procedures (15) and (16), equations (34) and (35) are simplified to

$$
\begin{aligned}
& \overline{\mathbf{x}}_{0 s}^{(1)}-\delta R_{2} \mathbb{S}_{0} \overline{\mathbf{x}}_{0 s}^{(2)}-\delta R_{2}\left\langle\mathbb{S}_{1} \widetilde{\mathbf{x}}_{1 \tau}^{(2)}\right\rangle=0, \\
& \overline{\mathbf{x}}_{0 s}^{(2)}-\delta R_{1} \mathbb{S}_{0} \overline{\mathbf{x}}_{0 s}^{(1)}-\delta R_{1}\left\langle\mathbb{S}_{1} \widetilde{\mathbf{x}}_{1 \tau}^{(1)}\right\rangle=0 .
\end{aligned}
$$

Expressions (36) still contain unknown functions $\left\langle\mathbb{S}_{1} \widetilde{\mathbf{x}}_{1 \tau}^{(2)}\right\rangle$ and $\left\langle\mathbb{S}_{1} \widetilde{\mathbf{x}}_{1 \tau}^{(1)}\right\rangle$, which can be determined from (39). Hence, by rewriting,

$$
\begin{aligned}
\delta R_{1}\left\langle\mathbb{S}_{1} \widetilde{\mathbf{x}}_{1 \tau}^{(1)}\right\rangle & =\delta R_{1}\left\langle\widetilde{\mathbf{r}}_{1 k} \frac{\partial \mathbb{S}_{0 i j}\left(\mathbf{r}_{0}\right)}{\partial x_{k}} \widetilde{\mathbf{x}}_{1 \tau}^{(1)}\right\rangle=\delta R_{1} \frac{\partial \mathbb{S}_{0 i j}\left(\mathbf{r}_{0}\right)}{\partial x_{k}}\left\langle\widetilde{\mathbf{r}}_{1 k} \widetilde{\mathbf{x}}_{1 \tau}^{(1)}\right\rangle \\
& =\delta R_{1} \frac{\partial \mathbb{S}_{0 i j}\left(\mathbf{r}_{0}\right)}{\partial x_{k}}\left\langle\left(\mathbf{x}_{1 k}^{(1)}-\mathbf{x}_{1 k}^{(2)}\right) \widetilde{\mathbf{x}}_{1 \tau}^{(1)}\right\rangle .
\end{aligned}
$$

The use of (32) and (33) into (37) yields to

$$
\begin{aligned}
& \delta R_{1}\left\langle\widetilde{\mathbb{S}}_{1} \widetilde{\mathbf{x}}_{1 \tau}^{(1)}\right\rangle=\delta \frac{m^{2} R_{1}^{3} \widehat{R}}{r_{0}^{2}} \mathbf{n}_{0 j} \overline{\mathbf{G}}_{i j}, \\
& \delta R_{2}\left\langle\mathbb{S}_{1} \widetilde{\mathbf{x}}_{1 \tau}^{(2)}\right\rangle=\delta \frac{m^{2} R_{2}^{3} \widehat{R}}{r_{0}^{2}} \mathbf{n}_{0 j} \overline{\mathbf{G}}_{i j},
\end{aligned}
$$

where $\overline{\mathbf{G}}_{i j}=\left\langle\widetilde{\mathbf{g}}_{i}^{\tau} \widetilde{\mathbf{g}}_{j}\right\rangle$ and $\widehat{R}=R_{2}^{2}-R_{1}^{2}$.

Substituting (38) into (36), keeping at most linear in $\delta$ terms, leads to

$$
\begin{aligned}
& \overline{\mathbf{x}}_{0 i s}^{(1)}=\delta \frac{m^{2} R_{2}^{3} \widehat{R}}{r_{0}^{2}} \mathbf{n}_{0 j} \overline{\mathbf{G}}_{i j}, \\
& \overline{\mathbf{x}}_{0 i s}^{(2)}=\delta \frac{m^{2} R_{1}^{3} \widehat{R}}{r_{0}^{2}} \mathbf{n}_{0 j} \overline{\mathbf{G}}_{i j} .
\end{aligned}
$$

The relationship between dual tensor $\overline{\mathbf{G}}_{i}$ and $\overline{\mathbf{G}}_{k l}$, see, e.g., [18], is defined as

$$
\overline{\mathbf{G}}_{i} \equiv 2 e_{i j k} \overline{\mathbf{G}}_{j k},
$$

where the notation $e_{i j k}=0,1,-1$, if any two of $i, j, k$ are the same, if $i, j, k$, is an even permutation of $1,2,3$, and if $i, j, k$ is an odd permutation of 1,2,3, respectively. Using (40), (39) can be written as

$$
\begin{aligned}
& \overline{\mathbf{x}}_{0 i s}^{(1)}=\delta \frac{m^{2} R_{2}^{3} \widehat{R}}{r_{0}^{3}}\left(\mathbf{r}_{0} \times \overline{\mathbf{G}}\right)_{i}, \\
& \overline{\mathbf{x}}_{0 i s}^{(2)}=\delta \frac{m^{2} R_{1}^{3} \widehat{R}}{r_{0}^{3}}\left(\mathbf{r}_{0} \times \overline{\mathbf{G}}\right)_{i} .
\end{aligned}
$$

The general vectorial notation of (41) is the solution of (11) written as

$$
\overline{\mathbf{x}}_{s}^{(v)}=\delta \frac{m^{2} R_{\mu}^{3} \widehat{R}}{r^{3}}(\mathbf{r} \times \overline{\mathbf{G}}), \quad \mu \neq v,
$$

where $\overline{\mathbf{x}}_{0 i s}^{(1)}$ and $r_{0}$ are replaced with $\overline{\mathbf{x}}_{s}^{(1)}$ and $r$. This is the main outcome of the paper.

From the main result (42), we obtain the relationship between the velocities of microspheres

$$
\overline{\mathbf{x}}_{s}^{(2)}=\lambda^{3} \overline{\mathbf{x}}_{s}^{(1)}, \quad \text { where } \lambda=\frac{R_{1}}{R_{2}} .
$$

Moreover, the vector form of the separation distance between the centres of microspheres is given by

$$
\mathbf{r}_{s}=\delta \frac{m^{2} \widehat{R}\left(R_{2}^{3}-R_{1}^{3}\right)}{r^{3}}(\mathbf{r} \times \overline{\mathbf{G}}) .
$$

This result shows that the two microspheres $\overline{\mathbf{x}}_{s}^{(1)}$ and $\overline{\mathbf{x}}_{s}^{(2)}$ move along a circular path (or along an arc of a circle) of radius $r$.

The result (42), which is due to the prescribed induced acceleration $\widetilde{\mathbf{g}}(\tau)$, has the following interesting cases:

(i) If the two microspheres are identical with $R_{1}=R_{2}$, or in the limit as $r \longrightarrow \infty$, the microspheres do not interact at all, then (42) becomes

$$
\overline{\mathbf{x}}_{s}^{(v)}=0
$$

which indicates that there is no translation motion for the system of two microspheres. Mathematically, it means that as the large separation, $r \longrightarrow \infty$, only the active sphere is moving on oscillatory motion along the direction of the applied external field, while the other nonconducting sphere remains stationary. However, when $R_{1}=R_{2}$, both spheres move on an oscillatory motion along the direction of the applied external field, which agrees with the findings of the classical studies of the motion of equal spheres in a viscous fluid at low Reynolds number, see, e.g., [19] and [5].

(ii) For $R_{1} \neq R_{2}$, multiplying both sides of (44) by $\mathbf{r}$ yields

$$
\mathbf{r} \cdot \mathbf{r}_{s}=\delta \frac{m^{2} \widehat{R}\left(R_{2}^{3}-R_{1}^{3}\right)}{r^{3}} \mathbf{r} \cdot(\mathbf{r} \times \overline{\mathbf{G}})=0,
$$

which leads to $\mathbf{r}_{s}=0$, and hence $\mathbf{r}$ is a constant. Physically, it means that trajectories of the microspheres cannot overlap each other.

It is important to note that the average velocity can be arranged by appropriate choice of the induced acceleration, $\widetilde{\mathbf{g}}(\tau)$. Let us consider a particular example 


$$
\widetilde{\mathbf{g}}(\tau)=\left[\begin{array}{c}
a \sin \tau \\
b \cos \tau \\
c \sin 2 \tau
\end{array}\right]
$$

where $a, b$, and $c$ are constants. The tilde-integration of (47) is required in calculating $\widetilde{\mathbf{g}}_{i}^{\tau} \widetilde{\mathbf{g}}_{k}$. Hence,

$$
\begin{aligned}
& \widetilde{\mathbf{g}}^{\tau}(\tau)=\left[\begin{array}{c}
-a \cos \tau \\
b \sin \tau \\
\left(\frac{-c}{2}\right) \cos 2 \tau
\end{array}\right], \\
& \widetilde{\mathbf{g}}_{i}^{\tau} \widetilde{\mathbf{g}}_{k}=\left[\begin{array}{ccc}
a^{2} \sin \tau \cos \tau & -a b \cos ^{2} \tau & -a c \sin 2 \tau \cos \tau \\
a b \sin ^{2} \tau & b^{2} \sin \tau \cos \tau & b c \sin 2 \tau \sin \tau \\
\frac{-a c}{2} \cos 2 \tau \sin \tau \frac{-b c}{2} \cos 2 \tau \cos \tau \frac{-c^{2}}{2} \sin 2 \tau \cos 2 \tau
\end{array}\right] .
\end{aligned}
$$

Using the averaging process (15), and the definition of dual tensor (40), we calculate $\overline{\mathbf{G}}_{i k}=\left\langle\widetilde{\mathbf{g}}_{i}^{\tau} \widetilde{\mathbf{g}}_{j}\right\rangle$,

$$
\begin{gathered}
\overline{\mathbf{G}}_{i k}=\frac{a b}{2}\left[\begin{array}{ccc}
0 & -1 & 0 \\
1 & 0 & 0 \\
0 & 0 & 0
\end{array}\right], \\
\overline{\mathbf{G}}=-a b\left[\begin{array}{l}
0 \\
0 \\
1
\end{array}\right] .
\end{gathered}
$$

Hence,

$$
\mathbf{r} \times \overline{\mathbf{G}}=\left[\begin{array}{c}
r_{1} \\
r_{2} \\
r_{3}
\end{array}\right] \times\left[\begin{array}{c}
0 \\
0 \\
-a b
\end{array}\right]=a b\left[\begin{array}{c}
-r_{2} \\
r_{1} \\
0
\end{array}\right]
$$

The substitution of (50) into (42) yields

$$
\overline{\mathbf{x}}_{s}^{(v)}=\delta \frac{m^{2} R_{\mu}^{3} \widehat{R}}{r^{3}} a b\left[\begin{array}{c}
-r_{2} \\
r_{1} \\
0
\end{array}\right] \text {. }
$$

Using the definition (1), we write (51) as

$$
\overline{\mathbf{x}}_{s}^{(\nu)}=\delta \frac{m^{2} R_{\mu}^{3} \widehat{R}}{r^{3}} a b\left[\begin{array}{c}
-\left(x_{2}^{(1)}-x_{2}^{(2)}\right) \\
\left(x_{1}^{(1)}-x_{1}^{(2)}\right) \\
0
\end{array}\right],
$$

which gives

$$
\overline{\mathbf{x}}_{s}^{(1)}=\left[\begin{array}{c}
x_{1 s}^{(1)} \\
x_{2 s}^{(1)} \\
x_{3 s}^{(1)}
\end{array}\right]=\delta A_{1}\left[\begin{array}{c}
-\left(x_{2}^{(1)}-x_{2}^{(2)}\right) \\
\left(x_{1}^{(1)}-x_{1}^{(2)}\right) \\
0
\end{array}\right], \overline{\mathbf{x}}_{s}^{(2)}=\left[\begin{array}{c}
x_{1 s}^{(2)} \\
x_{2 s}^{(2)} \\
x_{3 s}^{(2)}
\end{array}\right]=\delta A_{2}\left[\begin{array}{c}
-\left(x_{2}^{(1)}-x_{2}^{(2)}\right) \\
\left(x_{1}^{(1)}-x_{1}^{(2)}\right) \\
0
\end{array}\right],
$$


where $A_{1}=m^{2} R_{2}^{3} \widehat{R} a b / r^{3}$ and $A_{2}=m^{2} R_{1}^{3} \widehat{R} a b / r^{3}$ are constant. The above equations (53) can be written as the system of linear differential equations

$$
\begin{aligned}
& x_{1 s}^{(1)}=\delta A_{1}\left(x_{2}^{(2)}-x_{2}^{(1)}\right), \\
& x_{2 s}^{(1)}=\delta A_{1}\left(x_{2}^{(2)}-x_{2}^{(1)}\right), \\
& x_{3 s}^{(1)}=0, \\
& x_{1 s}^{(2)}=\delta A_{2}\left(x_{2}^{(2)}-x_{2}^{(1)}\right), \\
& x_{2 s}^{(2)}=\delta A_{2}\left(x_{2}^{(2)}-x_{2}^{(1)}\right), \\
& x_{3 s}^{(2)}=0 .
\end{aligned}
$$

This system of equations with four unknown functions (54) and (55) can be solved analytically using the elimination method to give

$$
\begin{aligned}
& x_{1 s}^{(1)}+B x_{2}^{(1)}=0, \\
& B x_{1}^{(1)}-x_{2 s}^{(1)}=0,
\end{aligned}
$$

where $B=\delta a b m^{2} \widehat{R}\left(R_{2}^{3}-R_{1}^{3}\right) / r^{3}$ is constant.

After differentiation, we obtain the system of equations

$$
\begin{aligned}
& x_{1 s s}^{(1)}+B^{2} x_{1}^{(1)}=0, \\
& x_{2 s s}^{(1)}+B^{2} x_{2}^{(1)}=0 .
\end{aligned}
$$

The related solutions of the first part of equation (57) are

$$
\overline{\mathbf{x}}^{(1)}=\left[\begin{array}{c}
c_{1} \cos (B s)+c_{2} \sin (B s) \\
c_{3} \cos (B s)+c_{4} \sin (B s) \\
c
\end{array}\right] .
$$

The slow time scale $s=t / \omega$ implies that in order to obtain physical dimensionless trajectory of the spheres, we have to multiply $\overline{\mathbf{x}}^{(2)}$ and $\overline{\mathbf{x}}^{(1)}$ by $1 / \omega$.

In this paper, we consider the motion defined by (11) with large $\omega$ where all the associated functions and its derivatives belong to class $O(1)$, see, e.g., [20], such that in the asymptotic limit as $\omega \longrightarrow \infty$,

$$
\begin{aligned}
\max |\widetilde{\mathbf{x}}| & \sim 1, \\
\max \left|\widetilde{\mathbf{x}}_{\tau}\right| & \sim 1, \\
\max \left|\widetilde{\mathbf{x}}_{\tau \tau}\right| & \sim 1, \\
\max |\mathbf{x}| & \sim \frac{1}{\omega}, \\
\max \left|\mathbf{x}_{t}\right| & \sim 1, \\
\max \left|\mathbf{x}_{t t}\right| & \sim \omega .
\end{aligned}
$$

Applying (59) and (60) into (58) gives

$$
\begin{aligned}
& c_{1}+c_{2}=a, \\
& c_{3}+c_{4}=b .
\end{aligned}
$$

Differentiate (58) with respect to $s$, and then applying (59) and (60) yields

$$
\begin{aligned}
& -B c_{1}+B c_{2}=1, \\
& -B c_{3}+B c_{4}=1 .
\end{aligned}
$$

Solving (61) and (62), we obtain

$$
\begin{aligned}
& c_{1}=\frac{a B-1}{2 B}, \\
& c_{2}=\frac{a B+1}{2 B}, \\
& c_{3}=\frac{b B-1}{2 B}, \\
& c_{4}=\frac{b B-1}{2 B} .
\end{aligned}
$$

Substituting (63) into (58) yields

$$
\overline{\mathbf{x}}^{(1)}=\left[\begin{array}{c}
\left(\frac{a B-1}{2 B}\right) \cos (B s)+\left(\frac{a B+1}{2 B}\right) \sin (B s) \\
\left(\frac{b B-1}{2 B}\right) \cos (B s)+\left(\frac{b B+1}{2 B}\right) \sin (B s)
\end{array}\right] .
$$

Similarly, we can derive the solution of second part of (57), and thus, we can show that

$$
\overline{\mathbf{x}}^{(2)}=\lambda^{3} \overline{\mathbf{x}}^{(1)} \text {. }
$$

Figures 3 and 4 show the trajectory (64) of the first sphere $\mathbf{x}^{(1)}$ and (65) of the second sphere $\mathbf{x}^{(2)}$ for different frequencies when the value of $\lambda$ is significantly different from 1. The trajectories indicate that the microspheres move in a circular path, which means the oscillatory external field drives the two microspheres to perform a circulation motion about this fulcrum, and each sphere moves in an arc like orbit through the fluid. Qualitative shape of the microspheres trajectories was found to be inversely proportional to the frequency; a similar result can also be found in [21] and [22]. Moreover, when $\lambda<1$, the distance traveled by the active sphere $\mathbf{x}^{(1)}$ is greater than the distance traveled by the nonconducting sphere $\mathbf{x}^{(2)}$, which means the distance traveled by the active sphere $\mathbf{x}^{(1)}$ gets longer as the radius of the sphere $\mathbf{x}^{(2)}$ gets bigger. 


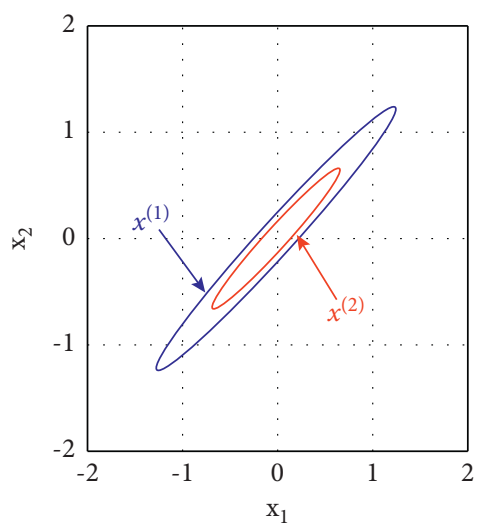

(a)

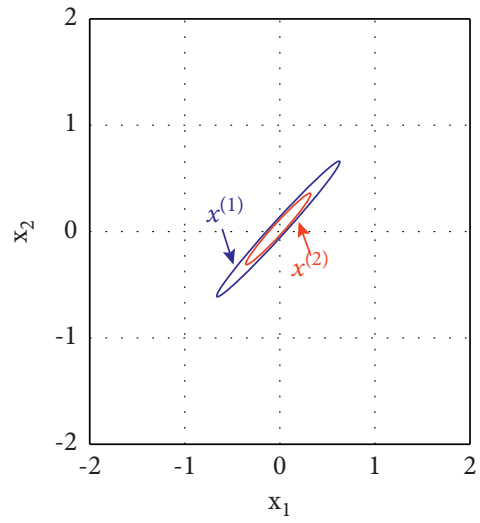

(b)

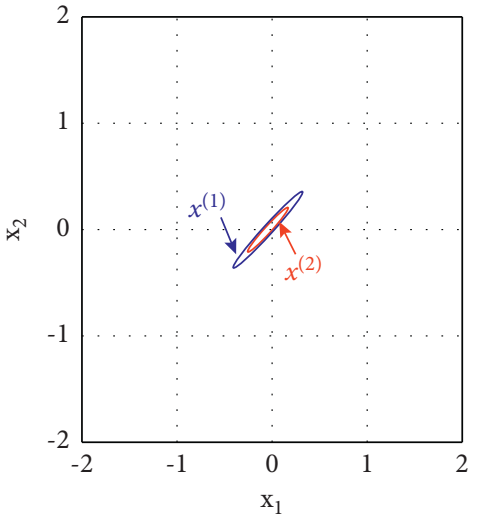

(c)

Figure 3: Trajectory of two microspheres $\mathbf{x}^{(1)}$ and $\mathbf{x}^{(2)}$ when $\lambda=0.7$ for different $\omega$. (a) $\omega=1.5$, (b) $\omega=3$, and (c) $\omega=5$.

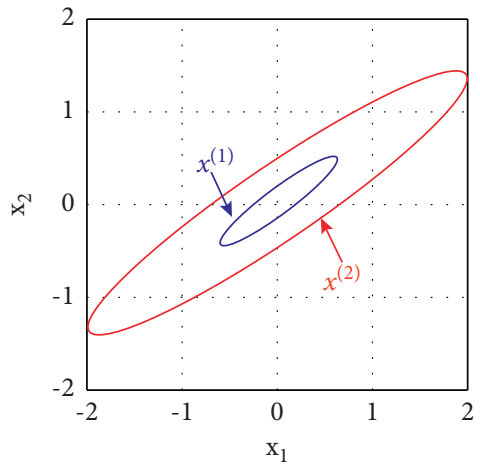

(a)

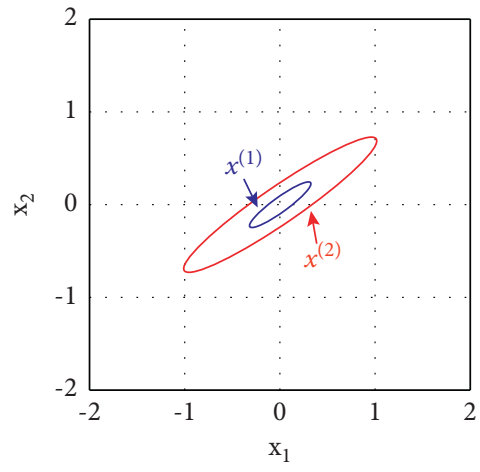

(b)

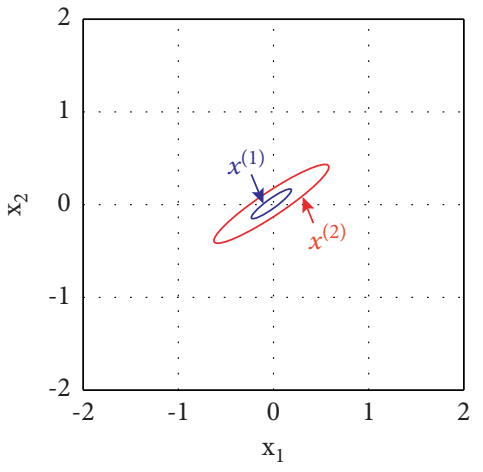

(c)

Figure 4: Trajectory of two microspheres $\mathbf{x}^{(1)}$ and $\mathbf{x}^{(2)}$ when $\lambda=1.5$ for different $\omega$. (a) $\omega=1.5$, (b) $\omega=3$, and (c) $\omega=5$.

\section{Conclusion}

In this paper, we studied analytically the motion of a system of two microspheres (one conducting and the other is not) in a Stokes flow driven by an external oscillator field. We constructed an asymptotic procedure with the dimensionless inverse frequency $\epsilon=1 / \omega$ and derived the average velocity of the system using the two-timing method. Our choice of slow time $s=\epsilon t$ and fast time $\tau=t / \epsilon$ led to a result that agrees with the experimental studies of an oscillating sphere in a viscous fluid, see, e.g., [23] and [21].

The result shows that the microspheres system moves in a circular motion with a fixed separation distance and travels a shorter distance as the frequency increases. We have demonstrated the ability of a conducting microsphere in a viscous fluid to influence the movement of other nonconducting microspheres in the neighboring area when an external oscillatory field is present. It is worth noting that the average velocities of the two microspheres and the vector of separation distance are given in the most general form, which can be applied to the ready-made formula by an appropriate choice of $\widetilde{\mathbf{g}}(\tau)$. The general procedure described here is likely applicable to other problems concerning the effect of microspheres interactions in viscous fluids that have the same properties and conditions of motion.

\section{Data Availability}

No data were used to support this study.

\section{Conflicts of Interest}

The authors declare that they have no conflicts of interest.

\section{Acknowledgments}

The author M. M. Al-Hatmi is grateful and would like to thank Prof. V. Vladimirov for many useful discussions.

\section{References}

[1] B. J. Nelson, I. K. Kaliakatsos, and J. J. Abbott, "Microrobots for minimally invasive medicine," Annual Review of Biomedical Engineering, vol. 12, no. 1, pp. 55-85, 2010.

[2] J. Happel and H. Brenner, Low Reynolds Number Hydrodynamics with Special Applications to Particulate Media, Springer Science \& Business Media, Berlin, Germany, 2012.

[3] H. J. Wilson, "An analytic form for the pair distribution function and rheology of a dilute suspension of rough spheres in plane strain flow," Journal of Fluid Mechanics, vol. 534, pp. 97-114, 2005. 
[4] E. Guazzelli and J. Hinch, "Fluctuations and instability in sedimentation," Annual Review of Fluid Mechanics, vol. 43, pp. 97-116, 2008.

[5] E. J. Hinch, "Sedimentation of small particles," in Disorder and Mixing, pp. 153-162, Springer, Berlin, Germany, 1988.

[6] E. M. Purcell, "Life at low Reynolds number," American Journal of Physics, vol. 45, no. 1, pp. 3-11, 1977.

[7] V. A. Vladimirov, "Dumbbell micro-robot driven by flow oscillations," Journal of Fluid Mechanics, vol. 717, 2013.

[8] M. S. Rizvi, A. Farutin, and C. Misbah, "Three-bead steering microswimmers," Physical Review, vol. 97, no. 2, Article ID 023102, 2018.

[9] R. Dreyfus, J. Baudry, and H. A. Stone, "Purcell's "rotator": mechanical rotation at low Reynolds number," The European Physical Journal B, vol. 47, no. 1, pp. 161-164, 2005.

[10] M. Belovs and A. Cēbers, "Ferromagnetic microswimmer," Physical review. E, Statistical, nonlinear, and soft matter physics, vol. 79, no. 5, Article ID 051503, 2009.

[11] A. D. Gilbert, F. Y. Ogrin, P. G. Petrov, and C. P. Wimlove, "Theory of ferromagnetic microswimmers," Quarterly Journal of Mechanics \& Applied Mathematics, vol. 64, no. 3, pp. 239-263, 2010.

[12] S. T. Chang, V. N. Paunov, D. N. Petsev, and O. D. Velev, "Remotely powered self-propelling particles and micropumps based on miniature diodes," Nature Materials, vol. 6, no. 3, pp. 235-240, 2007.

[13] P. Romanczuk, M. Bär, W. Ebeling, B. Lindner, and L. Schimansky-Geier, "Active brownian particles," The European Physical Journal - Special Topics, vol. 202, no. 1, pp. 1-162, 2012.

[14] V. A. Vladimirov, "Magnetohydrodynamic drift equations: from Langmuir circulations to magnetohydrodynamic dynamo?" Journal of Fluid Mechanics, vol. 698, pp. 51-61, 2012.

[15] J. Norton, "What was Einstein's principle of equivalence?" Studies In History and Philosophy of Science Part A, vol. 16, no. 3, pp. 203-246, 1985.

[16] L. Landau and E. Lifshitz, "Course of theoretical physics," in Fluid MechanicsElsevier, Amsterdam, The Netherlands, 1987.

[17] A. Nayfeh, Perturbation Methods, John Wiley \& Sons, New York, NY, USA, 1973.

[18] R. Aris, Vectors, Tensors and the Basic Equations of Fluid Mechanics, Courier Corporation, 2012.

[19] E. Guazzelli and J. F. Morris, A Physical Introduction to Suspension Dynamics, Cambridge University Press, Cambridge, UK, 2011.

[20] V. A. Vladimirov, "On vibrodynamics of pendulum and submerged solid," Journal of Mathematical Fluid Mechanics, vol. 7, no. 3, pp. S397-S412, 2005.

[21] G. Grosjean, M. Hubert, G. Lagubeau, and N. Vandewalle, "Realization of the najafi-golestanian microswimmer," Physical Review, vol. 94, no. 2, Article ID 021101, 2016.

[22] T. Lyubimova, D. Lyubimov, and M. Shardin, "The interaction of rigid cylinders in a low Reynolds number pulsational flow," Microgravity Science and Technology, vol. 23, no. 3, pp. 305-309, 2011.

[23] F. Box, K. Singh, and T. Mullin, "The interaction between rotationally oscillating spheres and solid boundaries in a Stokes flow," Journal of Fluid Mechanics, vol. 849, pp. 834859, 2018. 